\title{
AVALIAÇÃO DA SEDIMENTABILIDADE DE BIOMASSA GRANULAR E FLOCULENTA EM SISTEMAS DE LODO ATIVADO
}

\author{
E. V. M. SANTOS ${ }^{1}$, J. F. LIMA, H. A. SILVA FILHO, J. T. SOUZA e A. C. V. HAANDEL \\ Universidade Estadual da Paraíba - UEPB \\ elivania.ifce@yahoo.com.br ${ }^{1}$
}

Artigo submetido em novembro/2013 e aceito em janeiro/2014

DOI: http://dx.doi.org/10.15628/holos.2014.1916

\section{RESUMO}

A sedimentação é uma importante operação unitária no tratamento de esgotos, pois pode definir o desenvolvimento de projetos mais econômicos. A pesquisa visou avaliar a sedimentabilidade de biomassa aeróbia granular e floculenta. Foram montados dois sistemas de lodo ativado, um sistema University of Cape Town - UCT com características de lodo floculento e um reator em bateladas sequenciais com estratégias para a granulação aeróbia - RBSG. Com os resultados obtidos foi possível afirmar que o lodo granular obteve uma velocidade de sedimentação elevada $(35 \mathrm{~m} / \mathrm{h})$, sendo superior à velocidade considerada ideal pela literatura especializada (faixa de 6 a $10 \mathrm{~m} / \mathrm{h}$ ). Já o sistema UCT teve uma média de $7,9 \mathrm{~m} / \mathrm{h}$. Foi confirmado que o lodo granular do reator intermitente além da vantagem de maior acúmulo de sólidos no sistema e menor espaço construtivo, também é capaz de tratar mais esgoto por unidade de tempo quando comparado ao sistema contínuo com lodo floculento (UCT). Isso faz com que a escolha por sistemas granulares aeróbios se torne cada vez mais atrativa para o tratamento de efluentes de diferentes naturezas.

PALAVRAS-CHAVE: Lodo Ativado, Sedimentabilidade, Velocidade

\section{SETTLEABILITY EVALUATION OF BIOMASS FLOCCULENT AND GRANULAR ACTIVATED SLUDGE SYSTEMS}

\begin{abstract}
Sedimentation is an important unit operation in sewage treatment because it may define the development of more economical designs. The research aimed to evaluate the settleability of granular and flocculent aerobic biomass. Were mounted two activated sludge systems, one University of Cape Town - UCT with characteristics of flocculent sludge and sequencing batch reactor with strategies for aerobic granulation - RBSG. With the results we state that the granular sludge obtained a high sedimentation rate $(35 \mathrm{~m} / \mathrm{h})$, exceeding
\end{abstract}

the speed considered ideal by the literature (range 6-10 $\mathrm{m} / \mathrm{h}$ ). Have the UCT system had an average of $7.9 \mathrm{~m} / \mathrm{h}$. It was confirmed that the granular sludge of reactor intermittent addition to the advantage of higher accumulation of solids in the system and less constructive space, is also able to treat more wastewater per unit time compared to continuous systems with flocculent sludge (UCT). This makes the choice of aerobic granular systems becomes increasingly attractive for the treatment of effluents of different nature.

KEYWORDS: Activated Sludge, Settleability, Speed.. 


\section{INTRODUÇÃO}

Projetos racionais em Engenharia Ambiental e Sanitária podem ser definidos como sendo aqueles que permeiam parâmetros iniciais fundamentados na maximização dos efeitos desejados com a minimização dos custos de implantação e operação. Nesse contexto, é fundamental a obtenção de parâmetros de projeto cada vez mais realísticos e otimizados, garantindo assim a difusão de novas tecnologias (LIMA, 2013). No tratamento de águas residuárias, uma etapa fundamental após os processos de depuração dos poluentes, é a etapa da separação entre o lodo e a fração líquida, conhecida como sedimentação.

Segundo Van Haandel; Van der Lubbe (2012), dois atributos são essenciais para a qualificação de um lodo quanto à sedimentabilidade, atributos esses definidos na velocidade de sedimentação da biomassa e na capacidade de armazenar maior volume de lodo em um mesmo espaço (compressibilidade). Dessa forma, quanto mais rápido e mais compressível for o lodo, menor será o tempo de sedimentação aplicado e, em função da alta concentração de sólidos, maior será sua capacidade metabólica volumétrica na degradação dos poluentes. Paralelemente é possível também aplicar índices de qualidade (índices volumétricos de lodo, IVL) que podem definir de forma geral a qualidade do lodo.

São conhecidas duas formas básicas de conformação e arranjo dos organismos na ocasião da formação da biomassa dispersa, denominadas de flocos e grânulos. $O$ crescimento na forma de flocos é o mais conhecido e difundido desde o surgimento dos sistemas de lodo ativado. Contudo, Morgenroth et. al (1997) observou pela primeira vez a possibilidade de geração de lodo aeróbio granular, característica esta atribuída até então apenas ao lodo anaeróbio tratando grande carga orgânica, segundo Van Haandel; Lettinga, (1994).

Desde então, muitas pesquisas foram desenvolvidas e evidenciaram o melhor desempenho metabólico do lodo aeróbio granular face ao lodo floculento, em especial por conta da sua capacidade de limitar a difusão de gases ao seu interior, possibilitando a criação de ambientes aeróbios, anóxicos e anaeróbios no mesmo grânulo, favorecendo o desenvolvimento de diversos processos simultaneamente como, por exemplo, a nitrificação e desnitrificação simultânea em elevados teores de oxigênio dissolvido associado à remoção biológica de fósforo (KISHIDA, et al. 2010; LEW, et al. 2012; WU, et al. 2012; LIAO, et al. 2013; MORALES, et al. 2013; ZHONG, et al. 2013; ZHOU, et al. 2013).

Contudo, ainda são insipientes os estudos caracterizando as propriedades mecânicas desses lodos, sobretudo caracterizações quantitativas aplicando modelos matemáticos de sedimentabilidade que definem velocidade de sedimentação e capacidade de compressibilidade do lodo.

Nesse sentido, a presente pesquisa visou avaliar a sedimentabilidade do lodo de dois sistemas de lodo ativado em escala experimental, um sistema de fluxo contínuo do tipo University of Cape Town (UCT) e um Reator em Batelada Sequencial Granular (RBSG) que possuíam características diferentes quanto ao tipo de crescimento e conformação do lodo. 


\section{MATERIAL E MÉTODOS}

A presente pesquisa foi do tipo experimental visando a caracterização quantitativa e da qualidade de sedimentação de diferentes biomassas. Para tanto, foram realizados testes do Índice Volumétrico de Lodo (IVL) com base na avaliação dos dados em quatro diferentes referências: Von Sperling (2012), Van Haandel; Marais (1999), Fróes (1996) e Jordão; Pessoa (2011), bem como, testes da Velocidade de Sedimentação em Zona (VSZ) propostos por Vesilind (1968).

A sedimentabilidade de Vesilind foi avaliada em relação à velocidade de sedimentação da biomassa, definida como $V_{0}(\mathrm{~m} / \mathrm{h})$, e sobre sua capacidade de compressibilidade, definida na constante K (L/g). Para realizar os testes utilizou-se o método de White (1975) adaptado por van Haandel e Marais (1999) e para determinar as constantes usou-se a equação de Vesilind (1968).

Para gerar os lodos que foram usados nos testes de sedimentabilidade, foram confeccionados dois sistemas de lodo ativado, um sistema University of Cape Town (UCT, Figura 1a) em regime contínuo de alimentação e características de lodo floculento e um reator em bateladas sequenciais (RBSG, Figura 1 b) com estratégias para granulação conforme descrito em De Kreuk (2006).

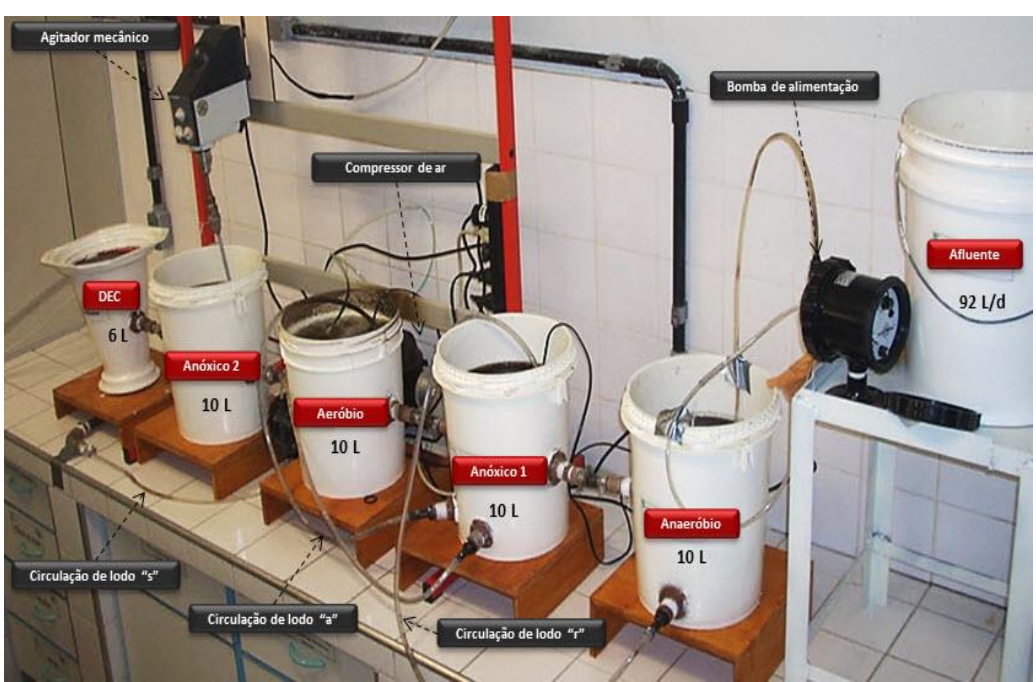

Figura 1a - Sistema UCT com 4 reatores (aeróbio, anóxico 1, aeróbio, anóxico 2 e decantador)

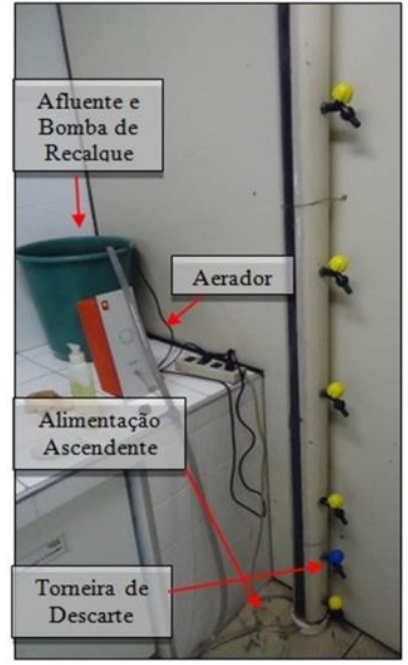

Figura 1b - Sistema RBSG (fluxo intermitente)

A água residuária usada para alimentação dos sistemas foi esgoto doméstico proveniente das instalações sanitárias e cantina de uma instituição de ensino (população flutuante em torno de 1.300 habitantes).

O sistema UCT possuía regime hidráulico contínuo, composto por 4 reatores e um decantador. O primeiro reator era anaeróbio, favorecendo a biodesfosfatação, o segundo reator era anóxico para desnitrificar e recebia nitrato (via recirculação de licor misto) do terceiro reator com ambiente aeróbio e que promovia a nitrificação. Por fim, seguia-se um último reator também anóxico para garantir melhor polimento na remoção de nitrato residual e findando em um decantador secundário que tinha a função de sedimentar o lodo para que o efluente saísse clarificado e que parte dessa biomassa retornasse para o sistema. 
O sistema RBSG seguiu um ciclo definido de 2 horas de hidrólise e 4 horas de aeração, com 15 minutos para a sedimentação sendo esse tempo reduzido para 3 minutos após a formação dos grânulos. A alimentação era feita simultaneamente ao momento de hidrólise.

Ambos os sistemas foram operados com idade de lodo de 20 dias e ampla disponibilidade de oxigênio dissolvido (OD $>3 \mathrm{mg} / \mathrm{L}$ ), em temperatura média de $27^{\circ} \mathrm{C}$. O tempo de partida foi superior a 3 meses em função da dificuldade da granulação aeróbia.

\subsection{Teste específico para determinação da velocidade de sedimentação em zona (VSZ)}

As constantes de sedimentabilidade modificam-se de acordo com a natureza do lodo, não podendo assim ser teoricamente estimada. Para avaliação das propriedades físicas, foi utilizada a metodologia descrita originalmente por White (1975) e Vesilind (1968), e aprimorada por Van Haandel; Marais (1999), Leitão (2004), Silva et al. (2008) e Souza (2011).

A equação de Vesilind relaciona a velocidade de sedimentação em zona com a concentração de sólidos em suspensão (Equação 1):

$\mathrm{V}=\mathrm{V}_{0} * e^{(-\mathrm{k} * \mathrm{xt})}$

Equação (1)

Onde

$\mathrm{V}$ : $\quad$ velocidade de sedimentação em zona em qualquer tempo (VSZ) em $\mathrm{m} / \mathrm{h}$;

$e$ : $\quad$ número ou constante de Euler ( $e=2,718281828 \ldots)$;

$\mathrm{X}_{\mathrm{t}}$ : concentração de sólidos em suspensão (gSST/L);

$\mathrm{V}_{0}, \mathrm{~K}$ : constantes de sedimentação em zona (velocidade de sedimentação inicial $(\mathrm{m} / \mathrm{h}$ ) e constante de compressibilidade (L/g)).

A velocidade de Sedimentação em Zona (VSZ) pode ser constatada em um decantador batelada, criado por White (1975). O equipamento é formado basicamente por um cilindro vertical transparente e graduado, onde se coloca o lodo (podendo ser agitado suavemente). Após a adição do lodo nota-se a separação da fase líquida (sobrenadante) e da biomassa que aos poucos vai sedimentando no interior do cilindro. Assim, ao se relacionar o deslocamento com o tempo, obtém-se a VSZ.

O equipamento utilizado no teste (Figura 2 ) desta pesquisa possuía uma fita métrica colada em sua superfície na vertical para que fosse perceptível o deslocamento do lodo $(\mathrm{em} \mathrm{cm})$ em função do tempo (min). Van Haandel; Marais (1999) aprimoraram o método, realizando várias baterias de teste com concentrações de lodo diferentes em cada um. Era retirado $25 \%$ do volume do lodo e preenchido com água a cada nova bateria, para obter uma diluição e assim uma concentração cada vez menor de sólido. $O$ teste desta pesquisa foi realizado em quatro baterias. 

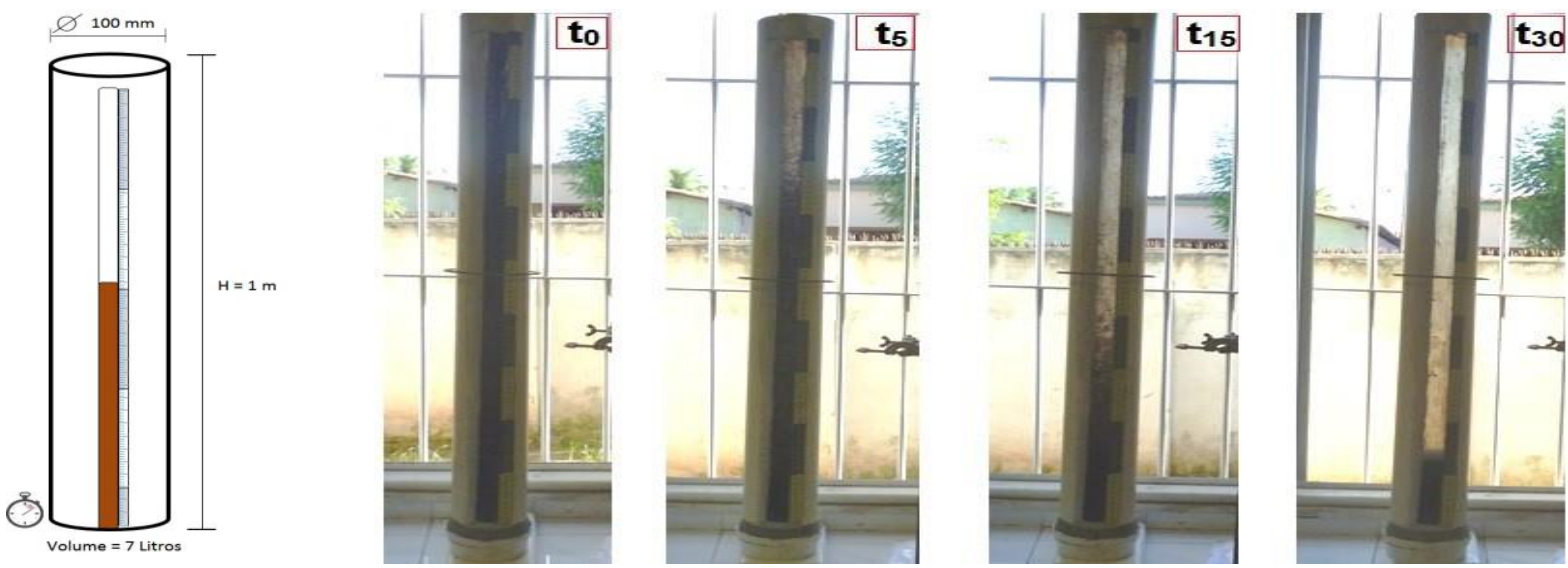

Figura 2: Cilindro usado para o teste de sedimentação (VSZ) e exemplo de perfil de deslocamento de lodo ao decorrer do tempo (t0, t5, t15 e t30).

Para determinar as constantes $\mathrm{K}$ e $\mathrm{V}_{0}$ plota-se um diagrama de escala semi-logarítmica (base e) da velocidade de sedimentação em zona em função da concentração de lodo (mg/L) segundo a equação de Vesilind (Equação 2). O valor da constante $\mathrm{K}$ é a declividade da reta e de $\mathrm{V}_{0}$ é o encontro da reta com o eixo das ordenadas.

A Figura 3 apresenta o gráfico com as curvas das quatro baterias de teste. A Figura 4 expressa os pontos que são selecionados para a composição da reta (apenas onde se identifica sedimentação).

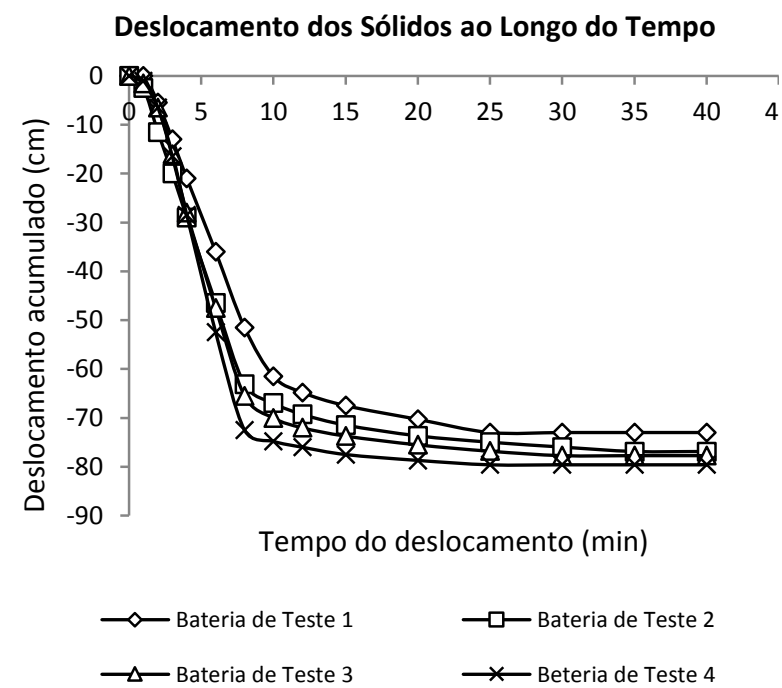

Figura 3: Curvas das quatro baterias do teste da VSZ

Fonte: Adaptado de Lima (2013)

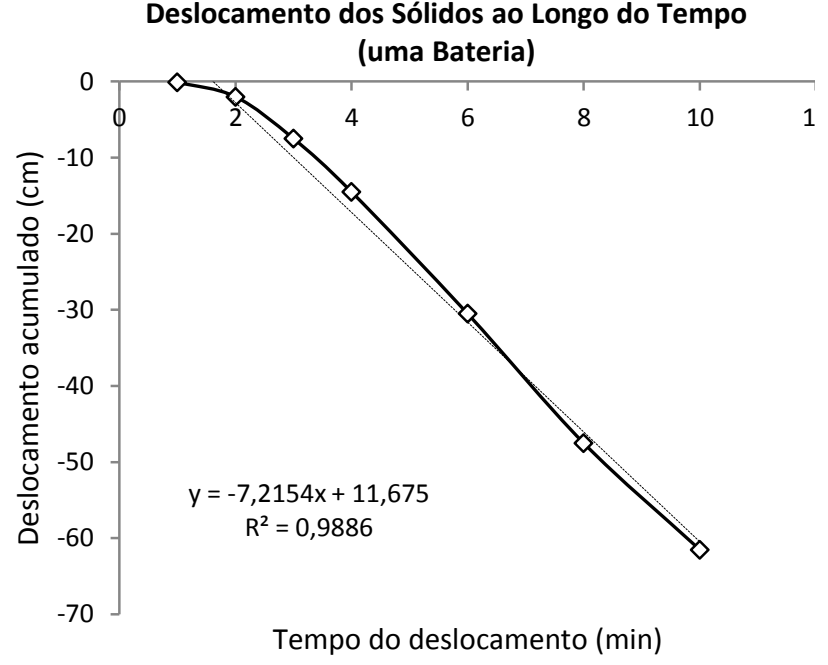

Figura 4: Curva de 1 bateria selecionada para linearização

(Efeito da compressibilidade removido).

Fonte: Adaptado de Lima (2013)

De posse desses dados ( $\mathrm{K}$ e $\mathrm{V}_{0}$ ), podemos então atribuir um aspecto qualitativo ao lodo em função da sua sedimentabilidade quando comparamos os valores obtidos aos de referência. Van Haandel; Marais (1999) classificam os valores de K e $V_{0}$ como Bom, Médio ou Ruim de acordo com a Tabela 1. 
Tabela 1: Classificação das constantes (K e Vo) por Van Haandel e Marais (1999)

\begin{tabular}{c|c|c|c}
\hline Parâmetros & BOM & MÉDIO & RUIM \\
\hline $\mathbf{V}_{\mathbf{0}}(\mathbf{m} / \mathbf{h})$ & 11 & 9,5 & 6,0 \\
\hline $\mathbf{K}(\mathbf{L} / \mathbf{g})$ & 0,31 & 0,36 & 0,46
\end{tabular}

2.2 Teste do Índice volumétrico de Lodo (IVL)

O IVL é um dos mais antigos testes para determinar a sedimentação, e também o mais utilizado na operação de ETE's, por ser simples a sua realização (MOHLMAN, 1934).

O teste consiste em determinar o volume que os sólidos ocupam após sedimentar em um cone Imhoff durante 30 minutos. Depois de determinar a concentração de sólidos inicial do lodo testado, calcula-se o volume ocupado por grama de sólidos em suspensão após ocorrer a sedimentação (VAN HAANDEL; MARAIS, 1999). Em cada bateria dos testes desta pesquisa VSZ era realizado o IVL, para obter valores diferentes e fazer uma comparação dos valores.

Segundo Von Sperling (1996) o IVL pode ser determinado pelo volume que ocupa $1 \mathrm{~g}$ de lodo após 30 min de sedimentação. O mesmo pode ser calculado como mostra a Equação 2:

$\mathrm{IVL}=\left(\mathrm{H}_{30} * 10^{6}\right) /\left(\mathrm{H}_{0} * \mathrm{SS}\right)$

Equação (2)

Onde:

IVL: Índice Volumétrico do Lodo $(\mathrm{ml} / \mathrm{g})$;

$\mathrm{H}_{30}$ : Altura da interface após 30 min de sedimentação $(\mathrm{m})$;

$\mathrm{H}_{0}$ : Altura da interface no instante $0(\mathrm{~m})$;

SS: Concentração de sólidos em suspensão (mg/L);

$10^{6}$ : Conversão de $\mathrm{mg}$ em g, e de $\mathrm{L}$ em $\mathrm{ml}$.

Cada autor interpreta o resultado do índice volumétrico do lodo de uma maneira, de acordo com sua prática ou estudos. As tabelas a seguir (Tabelas 2, 3, 4 e 5) expressam a interpretação ou citação de cada autor:

Tabela 2: Interpretação do índice por Van Haandel; Marais (1999)

\begin{tabular}{c|c}
\hline Sedimentabilidade & IVL \\
\hline Boa & $<50$ \\
Média & $50-65$ \\
Ruim & $>65$ \\
\hline
\end{tabular}

Tabela 3: Interpretação do índice por Von Sperling (2012)

\begin{tabular}{c|c}
\hline Sedimentabilidade & IVL \\
\hline Boa & $50-100$ \\
Média & $100-200$ \\
Má & $200-900$ \\
\hline
\end{tabular}


Tabela 4: Interpretação do índice por Jordão; Pessoa (2011)

\begin{tabular}{c|c}
\hline Sedimentabilidade & IVL \\
\hline Perfeita & $<50$ \\
Muito Boa & $50-100$ \\
Tolerável & $100-200$ \\
Má & $200-400$ \\
Praticamente & $>400$ \\
Impossível & \\
\hline
\end{tabular}

Tabela 5: Interpretação do índice por Froés

(1996)

\begin{tabular}{c|c}
\hline Sedimentabilidade & IVL \\
\hline Ótima & $50-100$ \\
Boa & $50-99$ \\
Média & $100-199$ \\
Ruim & $200-300$ \\
Péssima & $>300$ \\
\hline
\end{tabular}

\section{RESULTADOS E DISCUSSÃO}

Os dois sistemas operados geraram lodos com características diferentes (Figura 5), sendo o do RBSG granular e o do UCT floculento. Para determinar a sedimentabilidade dos mesmos, utilizou-se o método de White (1975) aperfeiçoado por Van Haandel; Marais (1999). A média do K (compressibilidade) e $V_{0}$ (velocidade) e do $r^{2}$ da equação de Vesilind estão apresentadas na Tabela 6.

Tabela 6 - Média das constantes de sedimentabilidade para cada lodo.
\begin{tabular}{c|c|c}
\hline \multirow{2}{*}{ Lodo } & \multicolumn{2}{|c}{ Média } \\
\cline { 2 - 3 } & $\mathbf{K}(\mathrm{L} / \mathrm{g})$ & $\mathbf{V}_{\mathbf{0}}(\mathrm{m} / \mathrm{h})$ \\
\hline UCT & 0,33 & 7,9 \\
RBS - Granular & 0,26 & 35,0 \\
\hline
\end{tabular}

Com os resultados obtidos foi possível afirmar que o lodo granular obteve uma melhor velocidade de sedimentação e compressibilidade, $35,0 \mathrm{~m} / \mathrm{h}$ e $0,26 \mathrm{~L} / \mathrm{g}$ respectivamente, isso se deu devido ao lodo ser em forma de grânulo e possuir maior densidade que o lodo floculento. Van Haandel; Marais (1999) sugerem que um lodo de boa sedimentabilidade possui valores de K=0,31 L/g e $V_{0}=11 \mathrm{~m} / \mathrm{h}$, valores esses superados pela característica da granulação.

O sistema UCT possui características de um lodo floculento, comum em sistemas de lodo ativado, mas sua velocidade apresentou-se pouco abaixo da referência considerada boa, 7,9 m/h, porém sua compressibilidade foi baixa $0,33 \mathrm{~L} / \mathrm{g}$ indicando assim que o mesmo ocupa mais espaço no decantador, pois quanto maior sua compactação menor espaço utilizado para decantação do lodo. 

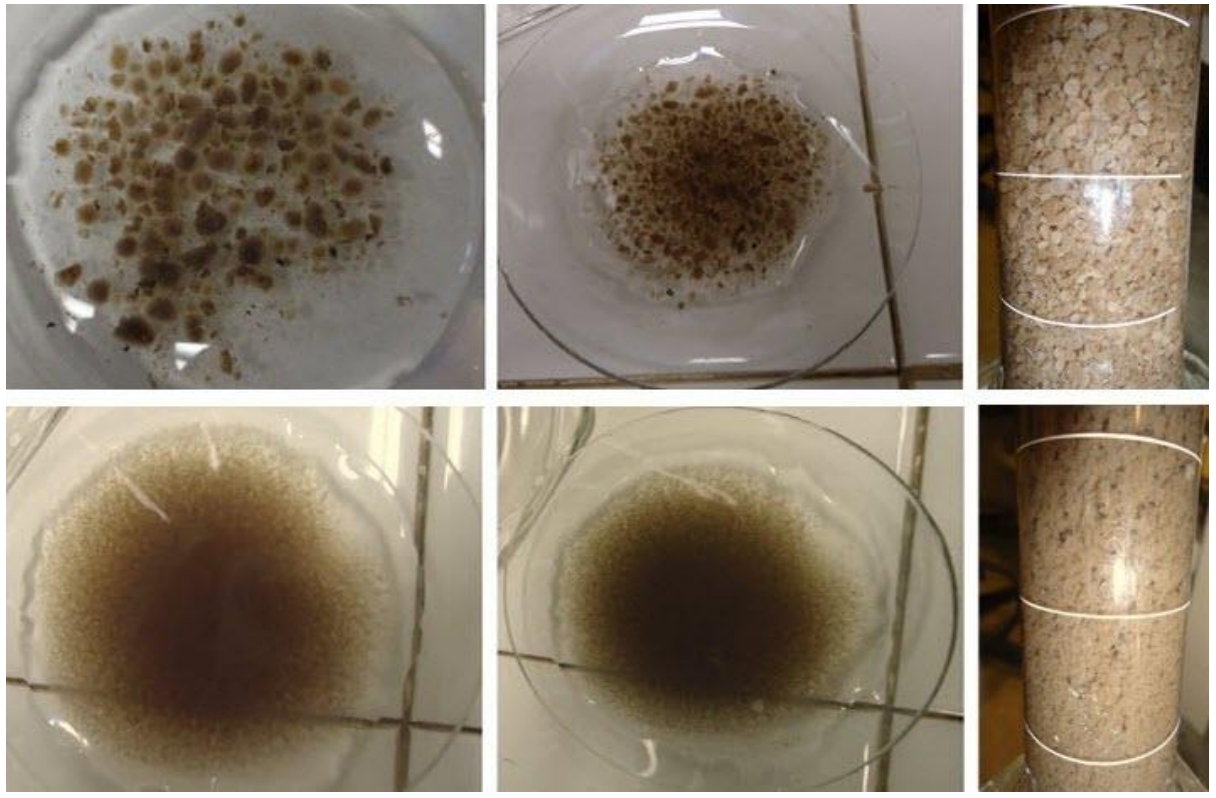

Figura 5: Tipos de lodos gerados nos sistemas; lodo granular (imagens superiores) do RBSG e lodo floculento (imagens inferiores) obtido no sistema UCT.

\subsection{Velocidade de Sedimentação em Zona (VSZ)}

Para determinar a sedimentabilidade do lodo gerado, utilizou-se o método de White (1975) modificado por Van Haandel; Marais (1999). A Tabela 7 apresenta os valores e a média das constantes $\mathrm{K}$ e $\mathrm{V}_{0}$ e do $\mathrm{r}^{2}$ da equação de Vesilind para os testes realizados.

Pela teoria de White (1975) e o teste de Vesilind (1968), um lodo com boa sedimentabilidade possui valores de $\mathrm{K}=0,31 \mathrm{~L} / \mathrm{g}$ e $\mathrm{V}_{0}=10 \mathrm{~m} / \mathrm{h}$, sendo valores abaixo dessa média considerados ruins (VAN HAANDEL; MARAIS 1999). O sistema UCT possui características de um lodo floculento, com isso sua velocidade média foi de 7,93 $\mathrm{m} / \mathrm{h}$, não se apresentando de acordo com o valor indicado como bom por Van Haandel; Marais (1999) podendo acarretar na saída de sólidos suspensos junto ao efluente tratado.

Todavia, a constante $\mathrm{K}$ foi igual a $0,33 \mathrm{~L} / \mathrm{g}$ indicando que o lodo possui uma boa compressibilidade, ou seja, que o mesmo ocupa menos espaço no decantador, pois quanto maior sua compactação menor espaço utilizado para decantação do lodo.

Já o sistema RBSG apresentou os valores das constantes de sedimentabilidade similares aos indicados como bons em Van Haandel; Marais (1999), sendo estes: constante da velocidade de sedimentação $35,0 \mathrm{~m} / \mathrm{h}$ e constante de compressibilidade igual a 0,26 L/g. 
Tabela 7: Valores de $\mathrm{K}$ e $\mathrm{V}_{0}$ dos testes realizados

\begin{tabular}{c|ccc}
\hline Teste UCT & $\boldsymbol{K}(\mathbf{L} / \boldsymbol{g})$ & $\boldsymbol{V}_{\boldsymbol{o}}(\boldsymbol{m} / \mathbf{h})$ & $r^{2}$ \\
\hline $\mathbf{1}$ & 0,47 & 10,90 & 0,83 \\
$\mathbf{2}$ & 0,38 & 8,38 & 0,98 \\
$\mathbf{3}$ & 0,15 & 6,78 & 0,96 \\
$\mathbf{4}$ & 0,45 & 8,08 & 0,97 \\
$\mathbf{5}$ & 0,18 & 7,61 & 1,00 \\
$\mathbf{6}$ & 0,17 & 6,03 & 0,97 \\
$\mathbf{7}$ & 0,51 & 7,75 & 0,99 \\
Média & $\mathbf{0 , 3 3}$ & $\mathbf{7 , 9 3}$ & $\mathbf{0 , 9 6}$ \\
\hline Teste $\boldsymbol{R B S G}$ & $\boldsymbol{K}(\mathbf{L} / \mathbf{g})$ & $\boldsymbol{V}_{\mathbf{0}}(\boldsymbol{m} / \mathbf{h})$ & $\boldsymbol{r}^{2}$ \\
\hline $\mathbf{1}$ & 0,27 & 34,92 & 0,95 \\
$\mathbf{2}$ & 0,38 & 31,98 & 0,98 \\
$\mathbf{3}$ & 0,28 & 36,78 & 0,96 \\
$\mathbf{4}$ & 0,25 & 34,07 & 0,99 \\
$\mathbf{5}$ & 0,18 & 32,71 & 0,93 \\
$\mathbf{6}$ & 0,17 & 36,53 & 0,94 \\
$\mathbf{7}$ & 0,31 & 37,75 & 0,97 \\
Média & $\mathbf{0 , 2 6}$ & $\mathbf{3 4 , 9 7}$ & $\mathbf{0 , 9 6}$ \\
\hline & & &
\end{tabular}

\section{2 Índice Volumétrico do Lodo (IVL)}

As Tabelas 8 e 9 apresentam médias dos resultados do Índice Volumétrico do Lodo das quatro baterias realizadas para cada referência estudada.

Tabela 8: Valores de IVL dos testes realizados para o sistema UCT

\begin{tabular}{c|ccccc}
\hline Testes UCT & $\begin{array}{c}\text { IVL } \\
(\mathbf{m} \mathbf{L} / \boldsymbol{g})\end{array}$ & $\begin{array}{c}\text { Van Haandel; } \\
\text { Marais (1999) }\end{array}$ & $\begin{array}{c}\text { Jordão; Pessoa } \\
(\mathbf{2 0 1 1 )}\end{array}$ & $\begin{array}{c}\text { Froés } \\
\text { (1996) }\end{array}$ & $\begin{array}{c}\text { Von Sperling } \\
(\mathbf{2 0 1 2})\end{array}$ \\
\hline $\mathbf{1}$ & 138,63 & Ruim & Tolerável & Média & Média \\
$\mathbf{2}$ & 167,65 & Ruim & Tolerável & Média & Média \\
$\mathbf{3}$ & 166,67 & Ruim & Tolerável & Média & Média \\
$\mathbf{4}$ & 111,75 & Ruim & Tolerável & Média & Média \\
$\mathbf{5}$ & 255,43 & Ruim & Tolerável & Média & Média \\
\hline
\end{tabular}

Com os valores de IVL obtidos nos testes foi possível avaliar pelas diferentes referências, uma qualificação geral da sedimentabilidade dos lodos dos sistemas. De acordo com Van Haandel; Marais (1999) o lodo testado em todos os experimentos com o sistema UCT obteve uma sedimentabilidade ruim, pois todos os valores passaram de $65 \mathrm{mg} / \mathrm{L}$, e os autores afirmam que o lodo com uma sedimentabilidade boa deve possuir um IVL igual ou menor que $50 \mathrm{mg} / \mathrm{L}$. Já para o sistema granular (RBSG) os autores consideram a sedimentabilidade de média a boa (Tabela 9). 
Tabela 9: Valores de IVL dos testes realizados para o sistema RBSG

\begin{tabular}{c|ccccc}
\hline Testes RBSG & $\begin{array}{c}\text { IVL } \\
(\mathbf{m} \mathbf{l} \boldsymbol{g})\end{array}$ & $\begin{array}{c}\text { Van Haandel; } \\
\text { Marais (1999) }\end{array}$ & $\begin{array}{c}\text { Jordão; Pessoa } \\
\text { (2011) }\end{array}$ & $\begin{array}{c}\text { Froés } \\
\text { (1996) }\end{array}$ & $\begin{array}{c}\text { Von Sperling } \\
\text { (2012) }\end{array}$ \\
\hline $\mathbf{1}$ & 52,63 & Média & Boa & Boa-Ótima & Boa \\
$\mathbf{2}$ & 47,65 & Boa & Perfeita & Boa-Ótima & Boa \\
$\mathbf{3}$ & 46,67 & Boa & Perfeita & Boa-Ótima & Boa \\
$\mathbf{4}$ & 51,75 & Média & Boa & Boa-Ótima & Boa \\
$\mathbf{5}$ & 45,43 & Boa & Perfeita & Boa-Ótima & Boa \\
\hline
\end{tabular}

Segundo Jordão; Pessoa (2011) o lodo do sistema UCT possui uma sedimentabilidade tolerável já que não ultrapassa 200 mg/L, mas assim como Van Haandel e Marais (1999) ele assegura que o lodo não deve ultrapassar $50 \mathrm{mg} / \mathrm{L}$ de IVL para possuir uma sedimentabilidade boa e não acarretar em problemas nas ETEs. Já o lodo do sistema granular, ainda de acordo com Jordão; Pessoa (2011) tem entre boa e perfeita sedimentabilidade.

Froés (1996) tem outra definição de qualidade para o mesmo lodo, pela sua conceituação para os valores obtidos neste estudo, identifica-se que o lodo do sistema UCT tem uma sedimentabilidade média, assim como em Von Sperling (2012), em que se encontra descrito de forma similar. A diferença principal entre as tabelas desses autores de qualidade de lodo quanto à sedimentabilidade, é que Froés sugere que o lodo não passe de $50 \mathrm{mg} / \mathrm{L}$ de IVL assim como os outros autores, mas Von Sperling (2012) estende esse valor a $100 \mathrm{mg} / \mathrm{L}$, dessa forma, o lodo testado não obteve a sedimentabilidade boa de acordo com a sua classificação.

Para Kishida et al. (2010) a sedimentação do lodo granular é maior que a de lodo floculento pelo fato de sua conformação ser especial e acumular possivelmente materiais poliméricos intracelulares, bem como fosfatos mineralizados. Já Wilderer; McSwain, (2004) consideram que a presença significativa de bactérias filamentosas na concentração da biomassa dispersa nos sistemas com crescimento de lodo floculento dificulta a sua sedimentabilidade em especial a sua compressibilidade.

\subsection{Velocidade de Sedimentação em Zona x Índice Volumétrico do Lodo}

A Tabela 10 expressa a comparação entre o teste da Velocidade de Sedimentação em Zona (VSZ) e o Índice Volumétrico do Lodo (IVL).

Tabela 10: Comparação - VSZ x IVL

\begin{tabular}{c|ccccc}
\hline Testes & VSZ & IVL 1 & IVL 2 & IVL 3 & IVL 4 \\
\hline Conceito UCT & Ruim & Ruim & Tolerável & Média & Média \\
Conceito RBSG & Bom & Boa & Perfeita & Boa-Ótima & Boa \\
\hline
\end{tabular}

*IVL 1: Van Haandel; Marais (1999); IVL 2: Jordão; Pessoa (2011); IVL 3: Froés (1996); IVL 4: Von Sperling (2012).

Ao se analisar a Tabela 10 é possível identificar que o teste do Índice Volumétrico de Lodo (IVL) que tem a melhor relação de qualidade comparada ao teste da Velocidade de Sedimentação em Zona (VSZ) é o IVL do Van Haandel; Marais (1999), pois para todos os seus resultados o lodo do sistema UCT obteve uma sedimentabilidade ruim, assim como foi constatado no teste da VSZ. 
O que foi finalmente confirmado quando se compararam os dados do sistema RBSG dos quatro autores sendo considerada uma sedimentabilidade boa. Neste último tipo de lodo os resultados dos outros autores foram compatíveis com o do teste de VSZ que pode ser indicativo de que alguns tipos de lodos (a exemplo do granular) possam se enquadrar aos índices qualitativos de IVL propostos nessas referências (JORDÃO; PESSOA, 2011; FROÉS, 1996; VON SPERLING, 2012).

Com os resultados obtidos também é possível afirmar que os testes de IVL propostos pelos autores: Jordão; Pessoa (2011), Froés (1996) e Von Sperling (2012) tornam-se menos acurados, pelo fato de que o teste da VSZ é um teste mais seguro que o IVL e que por considerar a concentração de sólidos é definitivamente mais confiável para aplicação de seus resultados em projetos.

\section{CONSIDERAÇÕES FINAIS}

Através dos resultados obtidos, conclui-se que o lodo que obteve melhor sedimentabilidade foi o lodo do RBSG, o granular com $35,0 \mathrm{~m} / \mathrm{h}$, isso pode ter ocorrido devido seu lodo ser em forma de grânulo, sendo assim mais denso que o outro; além de ter uma ótima velocidade, a compressibilidade também foi boa, pois esteve próximo do que a literatura cita como sedimentação boa.

O lodo do UCT mesmo sendo um lodo floculento, teve uma sedimentabilidade de $7,9 \mathrm{~m} / \mathrm{h}$ próximo de $10 \mathrm{~m} / \mathrm{h}$ (boa sedimentabilidade), isso se deu devido à operação do sistema. Ambos os sistemas foram bons em relação a sua compressibilidade (K).

De acordo com o teste da Velocidade de Sedimentação em Zona e sua constante de velocidade, o lodo do UCT testado não tem uma boa sedimentabilidade, como sugerem Van Haandel e Marais (1999), diferente do RBSG que apresentou excelente sedimentabilidade, superando os valores propostos como ideais por Van Haandel; Marais (1999).

Pelo teste do IVL de acordo com os quatro autores referenciados, o lodo do UCT testado também não obteve boa sedimentabilidade. Van Haandel; Marais (1999) afirmam que o lodo é ruim em termos de sedimentação; Jordão; Pessoa (2011) acreditam que o lodo tem uma sedimentação tolerável; Froés (1996) e Von Sperling (2012) citam que o lodo tem uma sedimentação média;

Em contraposição, para o lodo do RBSG, com base na qualificação dos autores citados, a sedimentabilidade se enquadra entre boa e perfeita.

Em comparação ao teste da Velocidade de Sedimentação em Zona e das referências do Índice Volumétrico do Lodo, a teoria de IVL mais indicada seria a de Van Haandel e Marais (1999), pois obteve resultado semelhante ao teste da VSZ, que sugere que o lodo tem uma sedimentação ruim para o UCT e boa para o RBSG.

Contudo, todos os resultados aproximaram-se em termos de qualificação dos lodos e confirma-se que a utilização do teste de IVL continua sendo bastante importante quando não se pode realizar a determinação das constantes de sedimentabilidade pelo teste da VSZ. 


\section{REFERÊNCIAS BIBLIOGRÁFICAS}

1. 1. DE KREUK, M.K.; VAN LOOSDRECHT, M.C.M. Formation of aerobic granules with domestic sewage. J. Environ. Eng. - ASCE 132(6). 2006.

2. 2. JORDÃO, E.P.; PESSOA, C.A. Tratamento de esgotos domésticos. $6^{\circ}$ ed. Associação Brasileira de Engenharia Sanitária. ABES. Rio de Janeiro 2011.

3. 3. KISHIDA, N.; KONO, A.; YAMASHITA, Y.; TSUNEDA, S. Formation of aerobic granular sludge in a continuous-flow reactor - control strategy for the selection of well-settling granular sludge. Journal of Water and Environment Technology 8(3): 251-258. 2010.

4. 4. LEITÃO, R.C. Robustness of UASB reactors treatig sewage under tropical conditions. Wageningen: Wageningen University, 160p. Tese de doutorado. 2004.

5. 5. LEW, B.; STIEF, P.; BELIAVSKI, M.; ASHKENAZI, A.; SVITLICA, O.; KHAND, A.; TARRE, S., DE BEER, D.; GREEN, M. Characterization of denitrifying granular sludge with and without the addition of external carbon source. Bioresource Technology 124(0): 413-420. 2012.

6. 6. LIAO, R.; SHENA, K.; LI, A.M.; SHI, P.; LI, Y.; SHI, Q.; WANGA, Z. High-nitrate wastewater treatment in an expanded granular sludge bed reactor and microbial diversity using 454 pyrosequencing analysis. Bioresource Technology 134(0): 190-197. 2013.

7. 7. LIMA, J.F.; CHAVES, J.R.; SANTOS, E.V.M.; SILVA FILHO, H.A. Avaliação da sedimentabilidade da biomassa em diferentes sistemas de tratamento. In: 70 Encontro Internacional das Águas. Recife, Pernambuco, 2013.

8. 8. MCSWAIN B.S.; IRVINE R.L.; WILDERER P.A. The influence of-settling time on the formation of aerobic granules, Water Sci. Technol., 50(10), 195-202. 2004.

9. 9. MOHLMAN, F.W. The sludge index. Sewage Wks J., Vol. 6, p.119-122; 54, 1934.

10. 10. MORALES, N.; FIGUEROA, M.; FRA-VÁZQUEZ, A.; VAL DEL RíO, A.; CAMPOS, J.L.; MOSQUERA-CORRAL, A.; MÉNDEZ, R. Operation of an aerobic granular pilot scale SBR plant to treat swine slurry. Process Biochemistry 48(8): 1216-1221. 2013.

11. 11. MORGENROTH, E.; SHERDEN, T.; VAN LOOSDRECHT, M.C.M.; HEIJNEN, J.J.; WILDERER P.A. Aerobic granular sludge in a sequencing batch reactor. Water Research 31(12): 3191-3194. 1997.

12. 12. SILVA, A.L; SILVA FILHO, H.A.; DERKS, Y.M.; CAVALCANTI, P.F.F.; VAN HAANDEL, A.C. Determinação das constantes de sedimentabilidade de sistemas de lodo ativado. XIII Simpósio Luso-Brasileiro de Engenharia Sanitária e Ambiental. ABES, Belém. 2008.

13. 13. SOUZA, F.A.F. Adaptação de lodo sanitário e industrial ao tratamento do vinhoto Dissertação (Mestrado em Engenharia Civil e Ambiental) - COENGE - Universidade Federal de Campina Grande - UFCG. Campina Grande - PB: UFCG. 2011.

14. 14. VAN HAANDEL, A.C.; LETTINGA, G. Tratamento Anaeróbio de Esgotos: Um Manual para Regiões de Clima Quente, Epgraf, Campina Grande, 240 p. 1994.

15. 15. VAN HAANDEL, A.C.; MARAIS, G. O comportamento do sistema de lodo ativado: teoria e aplicações para projetos e operações. Campina Grande - PB: Epgraf., 1999.

16. 16. VAN HAANDEL, A.C.; VAN DER LUBBE, J. Handbook biological wastewater treatment, design and optimization of activate sludge systems. 2012. 
17. 17. VESILIND, P.A. Theroretical considerations: desingn of prototype thickeners from batch settling test. Water and Sewage Works 1968.

18. 18. VON SPERLING, M. Princípios do tratamento de águas residuárias. Vol. 4. Lodos Ativados. 2. ed. Belo Horizonte: DESA-UFMG, v. 1. 428 p. 2002.

19. 19. WHITE M.J. Settling of activated sludge. Technical Report TR11, WRC Stevenhage - Reino Unido. 1985.

20. 20. WILDERER P.A.; MCSWAIN B.S. The SBR and its biofilm application potentials. Water Sci. Technol., 50(10), 1-10. 2004.

21. 21. WU, C.Y.; PENG Y.Z.; WANG, R.D.; ZHOU, Y.X. Understanding the granulation process of activated sludge in a biological phosphorus removal sequencing batch reactor. Chemosphere 86(8): 767-773. 2012.

22. 22. ZHONG, C.; WANG Y.; WANG Y.; LV, J.; LI, Y.; ZHU, J. High-rate nitrogen removal and its behavior of granular sequence batch reactor under step-feed operational strategy. Bioresource Technology 134(0): 101-106. 2013.

23. 23. ZHOU, M.; GONG, J.; YANG, C.; PU, W. Simulation of the performance of aerobic granular sludge SBR using modified ASM3 model. Bioresource Technology 127(0): 473-481. 2013. 